\title{
Comparison of Clinical Outcomes and Safety of Single-stage Bilateral and Unilateral Unicompartmental Knee Arthroplasty
}

\author{
(i) Fatih YILDIZ, iD Tunay ERDEN, id Gökçer UZER, id İbrahim TUNCAY
}

Bezmialem Vakıf University Faculty of Medicine, Department of Orthopedics and Traumatology, İstanbul, Turkey

\begin{abstract}
Objective: To evaluate the effectiveness and safety of bilateral Oxford medial unicompartmental knee arthroplasty (UKA) in the patients under a single anesthetic procedure.

Methods: Between October 2013 and December 2015, 225 knees of 181 (age 67.5 years) patients with at least two years of follow-up were evaluated. They were divided into two groups as unilateral group (group 1, $\mathrm{n}=137$ ) and one-stage simultaneous bilateral group (group 2, $\mathrm{n}=44$ ) for the comparisons. The outcome parameters were femoral and tibial component positions measured on the full-length radiographs, clinical outcomes using Oxford Knee Score (OKS), International Knee Documentation Committee Score (IKDC), patient reported satisfaction and complications.

Results: Between the groups, the mean follow-up periods $(\mathrm{p}=0.125)$, age $(\mathrm{p}=0.447)$, preoperative body mass index $(\mathrm{p}=0.288)$, OKS $(\mathrm{p}=0.314)$ and IKDC ( $\mathrm{p}=0.127)$ scores were not significantly different. Postoperatively, the mean flexion of the femoral component $(\mathrm{p}=0.544)$, posterior slope $(\mathrm{p}=0.511)$, varus-valgus angulation of the tibial components $(\mathrm{p}=0.358)$ were statistically similar between groups. Although the mean varus-valgus angulation of the femoral components $(\mathrm{p}=0.033)$ was statistically different between groups, the difference was too small to make clinical significance. The mean postoperative OKS $(\mathrm{p}=0.272)$ and IKDC $(\mathrm{p}=0.106)$ were similar between the groups. In group $1,21(16.0 \%)$ patients reported excellent, $91(69.5 \%)$ good and $4(3.1 \%)$ moderate satisfaction. Fifteen (11.5\%) patients reported non-satisfaction. In group 2, patients reported excellent satisfaction in $20(24.4 \%)$ knees, good in $50(61.0 \%)$ knees patients moderate in $2(2.4 \%)$ knees. Patients reported non-satisfaction in $10(12.2 \%)$ knees ( $>$ > 0.05$)$. Eight (5.8\%) complications in group 1 and, $3(3.4 \%)$ complications in group 2 were observed. The number of complications was not statistically different between the groups $(\mathrm{p}=0.535)$.

Conclusion: One-stage simultaneous bilateral Oxford medial UKA is a safe and effective method with acceptable complication rates compared to unilateral surgery.
\end{abstract}

Keywords: Unicondillar, unicompartmental, knee, artrhroplasty, bilateral

\section{Introduction}

Unicompartmental knee artroplasty (UKA) is a method with high patient satisfaction and successful results, which is used in the surgical treatment of medial joint osteoarthritis accompanied by complete thickness cartilage loss $(1,2)$. It is a less invasive procedure with shorter operation time (3), less blood loss, and without touching cartilage, bone, and ligaments in other parts of the knee, compared with total knee arthroplasty (TKA). In addition, stay in hospital is shorter and rehabilitation of the patients are faster with UKA (4-6). Although long-term results reveal that revision rate is a bit higher in UKA; UKA have important advantages such as lower morbidity and mortality rate and providing a more physiological joint compared with TKA $(1,7)$.
Address for Correspondence: Fatih YILDIZ, Bezmialem Vakıf University Faculty of Medicine, Department of Orthopedics and Traumatology, İstanbul, Turkey

E-mail: yildizfatih@hotmail.com ORCID ID: orcid.org/0000-0003-4475-5379

Cite this article as: Yıldız F, Erden T, Uzer G, Tuncay I. Comparison of Clinical Outcomes and Safety of Single-stage Bilateral and Unilateral Unicompartmental Knee Arthroplasty. Bezmialem Science 2019;7(1):47-51.

${ }^{\circ}$ Copyright 2019 by the Bezmiâlem Vakıf University

Bezmiâlem Science published by Galenos Publishing House.
Received: 17.01 .2018

Accepted: 12.03 .2018 
It is known that at least $20 \%$ of patients with knee arthroplasty due to gonarthrosis have disease in both joints and patients are admitted to the hospital for surgical treatment of other knees after a knee surgery $(3,8,9)$. The advantages of operating two knees in one surgery are decrease in treatment costs, shorter duration of hospitalization and shorter rehabilitation process (10-12). However, it is concerned that operating two knees in one surgery may prolong duration of the operation, increase the amount of bleeding and the need for transfusion, and complications, morbidity and mortality rate may be higher $(3,13)$.

This study was planned based on the hypothesis that bilateral medial unicondylar procedure is as reliable and effective as unilateral procedure. The aim of this study was to compare radiological prosthesis alignments, clinical functional scores, patient satisfaction and complications in patients with unilateral UKA and bilateral UKA in one surgery for medial joint osteoarthritis.

\section{Methods}

A total of 181 patients with primary medial joint osteoarthritis who underwent medial unicondylar knee arthroplasty between October 2013 and December 2015 due to the pain and functional limitation that did not improve despite conservative treatment were included in the study. Data were obtained retrospectively. Patients with at least two-year follow-up who had adequate documentation prior to and after the operation were included in the study. Patients who underwent UKA due to posttraumatic osteoarthritis or osteonecrosis and who had body mass index (BMI) higher than $40 \mathrm{~kg} / \mathrm{m}^{2}$ were excluded from the study. Patients who underwent bilateral UKA in different sessions were also excluded from the study. Patients were divided into two groups as unilateral UKA (group 1) and bilateral UKA in the same session (group 2).

In the preoperative period, the patients were asked about the localization of pain, the relationship between pain and activity and the presence of pain in front of knee and the presence of an underlying inflammatory disease. Height and weight of the patients were recorded to determine BMI. In physical examination, the knee range of motion was examined and valgus stress test for the presence of medial collateral ligament contracture was used to open the medial joint and to test the passive correctability of the varus deformity in the knee.

Informed consent was taken from all patients before the operation. Surgery was performed by two different surgeons working in the same orthopedic clinic under general or spinal anesthesia. Standard procedures were applied to all patients. About 30 minutes before incision, intravenous 2 grams cefazolin sodium was administered for prophylaxis against infection. The patients were prepared in arthroscopy position allowing knee movement 0-120 degrees on the sides that would be operated. After skin disinfection with betadine, patients were covered sterile. All patients underwent tourniquet as standard and the tourniquet pressure was inflated to be $300 \mathrm{mmHg}$. Capsulotomy was performed with approximately $8 \mathrm{~cm}$ medial parapatellar incision. After evaluating the stability of cartilage surfaces of lateral condyl, trochlea and patella, and anterior collateral ligament, the indication was also confirmed intraoperatively. In all patients, using the microplasty kit for the implantation of the cementless Oxford phase 3 prosthesis, appropriate tibial and femoral incisions were made and prostheses were placed. After the floors were properly closed, the tourniquet was opened. All patients walked with full load on the day after surgery and knee movements were started. Patients were discharged on the postoperative second day. Patients were called for follow-up at postoperative 3 weeks, 3 months, 1 year and later annually and were evaluated radiologically and functionally.

Preoperative and postoperative radiographic evaluations were performed with anterior-posterior and lateral knee radiographs and full-length leg radiographs while standing. Mechanical axis deviation (MAD) was measured on full-length leg radiograph before surgery; MAD, flexion of the femoral component, varusvalgus angulation of the femoral component, posterior tibial slope and varus-valgus angulation of the tibial component were measured on full-length leg radiograph after surgery.

The patients were evaluated with the International Knee Documentation Committee Score (IKDC) and the Oxford Knee Score (OKS) in the preoperative and postoperative controls. In the last control, patients were asked to choose one of the options that were very satisfied, satisfied, uncertain or not satisfied. Complications developed in the follow-ups were noted.

\section{Statistical Analysis}

Data were analyzed using the SPSS statistical program. Comparisons between groups were made using Mann-Whitney $\mathrm{U}$ and Pearson chi-square tests. A p value of $<0.05$ for $95 \%$ confidence interval was considered statistically significant.

\section{Results}

The mean age of the patients in group 1 consisting of 137 patients was 64.9 (44-86) years and the mean age of the patients in group 2 consisting of 44 patients with 88 knees was 66.1 (5181) years. A total of 255 knees were evaluated. There was no difference between the groups in terms of mean age $(\mathrm{p}=0.447)$, gender distribution $(\mathrm{p}=0.588)$, height $(\mathrm{p}=0.964)$ and weight $(\mathrm{p}=0.256)($ Table 1$)$.

Preoperative mean MAD decreased from $31.5 \mathrm{~mm}(0-86 \mathrm{~mm})$ to $16.1 \mathrm{~mm}(0-44 \mathrm{~mm})$ in group 1 after surgery (Table 2). Preoperative mean MAD decreased from $34.5 \mathrm{~mm}(0-90 \mathrm{~mm})$ to $15.3 \mathrm{~mm}(0-41 \mathrm{~mm})$ in group 2 after surgery. There was no difference in terms of decrease in mean MAD between groups $(\mathrm{p}=0.807)$. There was no statistically significant difference between groups in terms of postoperative prothesis alignment in radiographs such as flexion of the femoral component $(\mathrm{p}=0.544)$, posterior tibial slope $(\mathrm{p}=0.511)$ and varus-valgus angulation of the tibial component $(\mathrm{p}=0.358)$. However, varus-valgus angulation of the femoral component was measured as a mean of $10^{\circ}\left(1^{\circ}\right.$ $\left.19^{\circ}\right)$ in group 1 , whereas it was $9^{\circ}\left(0^{\circ}-21^{\circ}\right)$ in group $2(\mathrm{p}=0.033)$. Although there was a statistically significant difference between groups, a mean of $1^{\circ}$ difference was not clinically significant. 
There was no difference between groups in terms of functional evaluation with IKDC scores before $(\mathrm{p}=0.127)$ and after surgery $(\mathrm{p}=0.106)$ (Table 3). There was no difference between groups in terms of functional evaluation with OKS before $(\mathrm{p}=0.315)$ and after surgery $(\mathrm{p}=0.272)$. In terms of patient satisfaction; in group 1, $21(16.0 \%)$ patients were very satisfied, 91 (69.5\%) satisfied, $4(3.1 \%)$ uncertain and $15(11.5 \%)$ dissatisfied. In group 2, patients were asked about satisfaction for each knee individually and they were very satisfied for $20(24.4 \%)$ knees, satisfied for $50(61.0 \%)$ knees, uncertain for $2(2.4 \%)$ knees and dissatisfied for $10(12.2 \%)$ knees. There was no difference between groups in terms of satisfaction ratio $(\mathrm{p}>0.05)$.

\begin{tabular}{|c|c|c|c|}
\hline & $\begin{array}{l}\text { Group } 1 \\
(n=137)\end{array}$ & $\begin{array}{l}\text { Group } 2 \\
(n=44)\end{array}$ & $\mathrm{P}$ \\
\hline Mean follow-up (months) & 26.1 & 29.3 & 0.125 \\
\hline Mean age (years) & 64.9 & 66.1 & 0.447 \\
\hline $\begin{array}{l}\text { Mean preoperative BMI } \\
\left(\mathrm{kg} / \mathrm{m}^{2}\right)\end{array}$ & 32.0 & 33.1 & 0.288 \\
\hline Females & $110(80.3 \%)$ & $34(77.3 \%)$ & 0.617 \\
\hline Males & $27(19.7 \%)$ & $10(22.7 \%)$ & 0.351 \\
\hline
\end{tabular}

Table 2. Radiological evaluation of leg alignments and implant placements

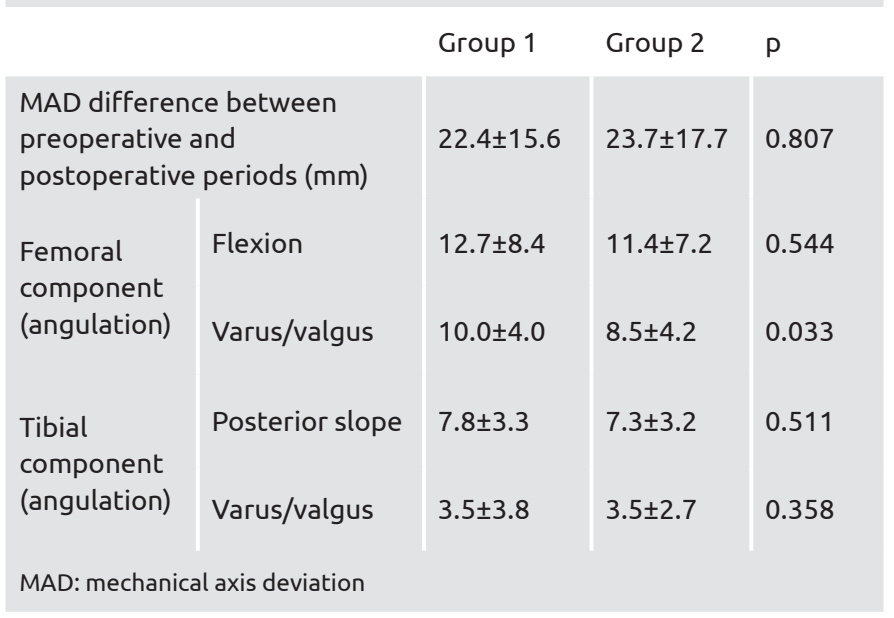

Table 3. Functional results before and after surgery

\begin{tabular}{|c|c|c|c|}
\hline & Group 1 & Group 2 & $\mathrm{p}$ \\
\hline OKS before surgery & $26.9 \pm 2.4$ & $26.6 \pm 1.9$ & 0.314 \\
\hline OKS after surgery & $38.5 \pm 2.6$ & $39.6 \pm 5.2$ & 0.272 \\
\hline IKDC before surgery & $38.6 \pm 6.1$ & $37.3 \pm 4.8$ & 0.127 \\
\hline IKDC after surgery & $70.3 \pm 7.5$ & $71.8 \pm 7.8$ & 0.106 \\
\hline
\end{tabular}

In group 1, 8 (5.8\%) patients and in group 2, $3(3.4 \%)$ patients and a total of $11(4.9 \%)$ patients had complications. There was no statistically significant difference between the groups in terms of complication $(\mathrm{p}=0.535)$. Polyethylene liner dislocation was encountered in 3 patients; 2 spontaneously in group 1 and 1 due to trauma in group 2 . In one patient, a thicker polyethylene liner was placed and in two patients, revision was made with primary knee prosthesis. In one patient, loosening due to early and severe osteolysis was observed around the implants and hypersensitivity was detected against cobalt and it was revised with primary TKA produced from oxinium and titanium. In one patient, in follow-up, varus collapse was encountered and was revised with knee prosthesis. Three patients were revised with primary knee arthroplasty in the first year due to pain of unknown. In group 2, acute prosthesis infection was seen and was treated by irrigation and debridement in one patient. Polyethylene liner dislocation was encountered in two patients, a thicker polyethylene liner was placed in one patient and revision was made with primary total knee prosthesis in the other patient.

\section{Discussion}

It is known that duration of anesthesia, hospitalization and rehabilitation are shorter in bilateral TKA or UKA application in the same session and it is also more economical for the patients and health system compared with bilateral TKA or UKA application in different session (11,14-16). However, there are also those who claim that the complication rates are higher $(17,18)$. In this retrospective study, clinical and radiological results of the patients with medial joint osteoarthritis in whom unilateral UKA and bilateral UKA in the same session were performed, were evaluated and compared with each other. Thus, it was aimed to see whether the risk of complications and bad clinical results were increased in bilateral UKA application. According to our findings; the radiographic and functional results and satisfaction of the patients with at least two years follow-up who had similar age, gender distribution, BMI and functional scores before surgery, were similar in those who underwent unilateral UKA and who underwent bilateral UKA. More importantly, the complication rates in patients with bilateral UKA were not higher than in those with unilateral UKA. For this reason, bilateral UKA should not be avoided in appropriate patients with indication to reduce treatment costs, to reduce patients' admission to hospital and to complete all rehabilitation process at once. In a similar study by Romagnoli et al. (3), complication rate and revision need of 220 patients with bilateral UKA in the same session and 347 patients with unilateral UKA were evaluated at the end of at least two years follow-up. Although it was found that blood loss and allogenic blood transfusions rates were higher in the patients treated with bilateral UKA, complication and revision rates were similar between groups. In that study, although patients underwent surgery without using tourniquet, intravenous or intraarticular tranexamic acid was not administered and although they were asymptomatic patients, patients with hemoglobin values below $8 \mathrm{mg} / \mathrm{dL}$ received transfusion which could explain the relatively high blood loss and transfusion rates in the bilateral UKA group (11). 
Similar to our results; complication $(3.5 \%$ vs $3 \% ; \mathrm{p}=0.83)$ and revision rates (approximately $1 \%$ in both groups; $\mathrm{p}=0.27$ ) were similar in patients treated with bilateral and unilateral UKA in the study by Romagnoli et al. (3). Complication rate was 5.8\% in group 1 and $3.4 \%$ in group 2 in our study. The lower revision rates in the series of Romagnoli et al. (3) can be explained by the fact that they had a very high number of patients (more than 2500) and had more experience and that single surgeon's surgical series were examined. In our series, although the revision rate in unilateral UKA was higher than expected, there was no statistically significant difference between groups $(\mathrm{p}=0.535)$. However, regardless of surgical technique, revision was made in one patient due to metal allergy and in three patients due to "pain of unknown" in whom radiographic and physical examinations were normal; which could explain the relative high revision rate in this group (19).

In a study that assessed the safety of bilateral UKA in same session by searching early postoperative complications, no major complication was found in patients who underwent bilateral UKA in different sessions, however major complications (deep vein thrombosis in 10 patients and myocardial infarction in one patient) were observed in $8.2 \%$ of the patients who underwent bilateral UKA in same session $(\mathrm{p}=0.005)(13)$. Therefore, it was recommended to be careful when applying bilateral UKA in the same session. In our study, no symptomatic deep vein thrombosis, cardiac or neurological complaints were encountered in any patient.

When we examined our radiographic results, we saw that the implants were placed within the desired and acceptable limits in both patients with bilateral and unilateral UKA. Although the placement of the implants in the left and right knee of the same patients was not compared, we think that bilateral UKA procedure does not result in poor implant placement.

A significant improvement was observed in functional results such as IKDC and OKS in both groups in the postoperative period compared with the preoperative period. OKS was above 39 points in both groups in the postoperative period in our study. Mohammad et al. (20) reported an average of 40 points in OKS in 10-year follow-up in a recent meta-analysis of more than 8.000 patients with UKA.

\section{Study Limitations}

Having a retrospective design is a limitation of our study. Comparison of similar patient populations in prospective studies may give more accurate results. In addition, the fact that bilateral UKA applications in the same session and different sessions were not compared in our study, which is another limitation of the study. There was low number of patients, short duration of follow-up and no evaluation with patient satisfaction scale in our study, which are other limiting factors. The number of publications containing large series is also very low in the literature. There was no comparison between groups in terms of bleeding volume, blood replacement need, hospitalization period and total treatment costs in this study, which is also a limitation of the study.

\section{Conclusion}

Although the number of patients was limited, the data of our study showed that bilateral UKA was as safe as unilateral UKA, and that there was no difference between bilateral and unilateral UKA in terms of patient satisfaction, functional and radiographic results, complication and revision rates.

\section{Ethics}

Ethics Committee Approval: Retrospective study.

Informed Consent: Informed consent was taken from all patients before the operation.

Peer-review: Externally peer-reviewed.

\section{Authorship Contributions}

Surgical and Medical Practices: F.Y., G.U., İ.T., Concept: F.Y., İ.T., Design: F.Y., G.U., Data Collection or Processing: F.Y., T.E., Analysis or Interpretation: F.Y., G.U., İ.T., T.E., Literature Search: F.Y., T.E., Writing: F.Y., T.E.

Conflict of Interest: No conflict of interest was declared by the authors.

Financial Disclosure: The authors declared that this study received no financial support.

\section{References}

1. Goh GS, Bin Abd Razak HR, Tay DK, Chia SL, Lo NN, Yeo SJ. Unicompartmental Knee Arthroplasty Achieves Greater Flexion With No Difference in Functional Outcome, Quality of Life, and Satisfaction vs Total Knee Arthroplasty in Patients Younger Than 55 Years. A Propensity Score-Matched Cohort Analysis. J Arthroplasty 2018;33(2):355-61.

2. Hanssen AD, Stuart MJ, Scott RD, Scuderi GR. Surgical options for the middle-aged patient with osteoarthritis of the knee joint. Instr Course Lect 2001;50:499-511.

3. Romagnoli S, Zacchetti S, Perazzo P, Verde F, Banfi G, Vigano M. Onsets of complications and revisions are not increased after simultaneous bilateral unicompartmental knee arthroplasty in comparison with unilateral procedures. Int Orthop 2015;39:871-7.

4. Pandit H, Jenkins C, Barker K, Dodd CA, Murray DW. The Oxford medial unicompartmental knee replacement using a minimallyinvasive approach. J Bone Joint Surg Br 2006;88:54-60.

5. Cross MB, Berger R. Feasibility and safety of performing outpatient unicompartmental knee arthroplasty. Int Orthop 2014;38:443-7.

6. Pietschmann MF, Wohlleb L, Weber P, Schmidutz F, Ficklscherer A, Gulecyuz MF, et al. Sports activities after medial unicompartmental knee arthroplasty Oxford III-what can we expect? Int Orthop 2013;37:31-7.

7. Kim MS, Koh IJ, Choi YJ, Lee JY, In Y. Differences in PatientReported Outcomes Between Unicompartmental and Total Knee Arthroplasties: A Propensity Score-Matched Analysis. J Arthroplasty 2017;32:1453-9.

8. Meehan JP, Danielsen B, Tancredi DJ, Kim S, Jamali AA, White $\mathrm{RH}$. A population-based comparison of the incidence of adverse 
outcomes after simultaneous-bilateral and staged-bilateral total knee arthroplasty. J Bone Joint Surg Am 2011;93:2203-13.

9. Sayeed SA, Sayeed YA, Barnes SA, Pagnano MW, Trousdale RT. The risk of subsequent joint arthroplasty after primary unilateral total knee arthroplasty, a 10-year study. J Arthroplasty 2011;26:842-6.

10. Berend KR, Morris MJ, Lombardi AV. Unicompartmental knee arthroplasty: incidence of transfusion and symptomatic thromboembolic disease. Orthopedics 2010;33(Suppl 9):8-10.

11. Chen JY, Lo NN, Jiang L, Chong HC, Tay DK, Chin PL, et al. Simultaneous versus staged bilateral unicompartmental knee replacement. Bone Joint J 2013;95-B:788-92.

12. Reuben JD, Meyers SJ, Cox DD, Elliott M, Watson M, Shim SD. Cost comparison between bilateral simultaneous, staged, and unilateral total joint arthroplasty. J Arthroplasty 1998;13:172-9.

13. Chan WC, Musonda P, Cooper AS, Glasgow MM, Donell ST, Walton NP. One-stage versus two-stage bilateral unicompartmental knee replacement: a comparison of immediate post-operative complications. J Bone Joint Surg Br 2009;91:1305-9.

14. Hersekli MA, Akpinar S, Ozalay M, Ozkoc G, Uysal M, Cesur N, et al. [A comparison between single- and two-staged bilateral total knee arthroplasty operations in terms of the amount of blood loss and transfusion, perioperative complications, hospital stay, and costeffectiveness]. Acta Orthop Traumatol Turc 2004;38:241-6.
15. Hutchinson JR, Parish EN, Cross MJ. A comparison of bilateral uncemented total knee arthroplasty: simultaneous or staged? J Bone Joint Surg Br 2006;88:40-3.

16. Powell RS, Pulido P, Tuason MS, Colwell CW, Ezzet KA. Bilateral vs unilateral total knee arthroplasty: a patient-based comparison of pain levels and recovery of ambulatory skills. J Arthroplasty 2006;21:642-9.

17. Yoon HS, Han CD, Yang IH. Comparison of simultaneous bilateral and staged bilateral total knee arthroplasty in terms of perioperative complications. J Arthroplasty 2010;25:179-85.

18. Stefansdottir A, Lidgren L, Robertsson O. Higher early mortality with simultaneous rather than staged bilateral TKAs: results from the Swedish Knee Arthroplasty Register. Clin Orthop Relat Res 2008;466:3066-70.

19. Baker PN, Petheram T, Avery PJ, Gregg PJ, Deehan DJ. Revision for unexplained pain following unicompartmental and total knee replacement. J Bone Joint Surg Am 2012;94:e126.

20. Mohammad HR, Strickland L, Hamilton TW, Murray DW. Long-term outcomes of over 8,000 medial Oxford Phase 3 Unicompartmental Knees-a systematic review. Acta Orthop 2017:1-7. 